\title{
Peritoneal Macrophage
}

National Cancer Institute

\section{Source}

National Cancer Institute. Peritoneal Macrophage. NCI Thesaurus. Code C12566.

A differentiated macrophage that resides in the serosal peritoneal membrane and plays an important role in the defense against peritoneal cavity infections. 